\title{
eJRIEPS
}

Ejournal de la recherche sur l'intervention en éducation physique et sport

$15 \mid 2008$

Varia

\section{Physique et sport : Alain Durey et 20 ans de recherches en technologie des APS}

\section{Philippe Varrin}

\section{OpenEdition}

Journals

Édition électronique

URL : https://journals.openedition.org/ejrieps/5823

DOI : 10.4000/ejrieps.5823

ISSN : 2105-0821

Éditeur

ELLIADD

\section{Référence électronique}

Philippe Varrin, "Physique et sport : Alain Durey et 20 ans de recherches en technologie des APS », eJRIEPS [En ligne], 15 | 2008, mis en ligne le 01 juillet 2008, consulté le 03 mai 2021. URL : http:// journals.openedition.org/ejrieps/5823 ; DOI : https://doi.org/10.4000/ejrieps.5823

\section{(c) (1)}

La revue eJRIEPS est mise à disposition selon les termes de la Creative Commons Attribution 4.0 International License. 
Physique et sport : Alain Durey et 20 ans de recherches en technologie des APS

Philippe Varrin

Technicien de recherche et formation, GHDSO, EA 1610,

Université Paris-Sud 11 et UMR STEF, ENS Cachan

Résumé :

Associé pendant 20 ans à ses recherches, mon propos se veut un témoignage sur les apports qu'Alain Durey a permis dans le domaine des activités physiques et sportives, tant au niveau de la transformation des pratiques que de la formation.

Ma collaboration aux recherches menées par Alain Durey s'est étendue sur une période de 20 ans. Commencée à l'ENS de Saint-Cloud au début des années quatre-vingt, elle s'est poursuivie à l'Université Paris 11 entre 1987 et 1991. Enfin, c'est à l'ENS de Cachan, qu'elle s'est achevée.

Cette relation professionnelle et amicale a permis de mesurer toute la richesse humaine que détenait Alain Durey. Tous ceux qui ont eu l'opportunité de travailler avec lui ont pu constater son extrême gentillesse, sa disponibilité, mais aussi son très grand professionnalisme. Alain Durey apportait la convivialité dans les groupes de recherche qui se constituaient mais aussi sa très grande rigueur scientifique.

Agrégé de physique, Alain Durey s'était intéressé initialement à l'usage des ordinateurs comme outils de laboratoire. Le mariage de la physique et de l'ordinateur ainsi que son très grand intérêt pour le sport ont fait de lui un spécialiste de la modélisation et de la simulation des "gestes " sportifs. Certains se souviennent peut-être encore qu'il avait simulé à l'ordinateur le fameux coup franc de Michel Platini en 1982 contre les Pays-Bas. La présentation des résultats de quelques recherches, illustre la démarche et les contributions d'Alain Durey dans le domaine des APS.

\section{La démarche initiale}

L'étude de tout geste sportif se base sur les principes de la physique, et la démarche initiale d'Alain Durey a été de montrer les possibilités de la physique scolaire (niveau $L d u$ cycle universitaire) à répondre aux questions posées par des acteurs extérieurs à l'environnement scolaire et en particulier ceux du monde sportif. 
Par l'élaboration de modèles physiques, leurs. mises au point (mise en place des dispositifs expérimentaux de recueils de données) et leurs validations (confrontation et ajustement du modèle élaboré à la réalité), des réponses étaient données sur divers aspects des pratiques sportives.

\section{De nouveaux outils méthodologiques}

Sur le plan de la méthodologie d'analyse du mouvement, des techniques ont été développées qui ont contribué au développement d'un logiciel intégré de digitalisation et d'analyse de données:

Prises de vues cinéma (caméras rapides de 500 images/sec à 3000 images/sec - figure 1), et vidéo (normale, rapide, "stroboscopique ") faites sur le terrain, parfois dans des conditions extrêmes (figure 2).

Réalisation d'un module de calcul, permettant la reconstitution en 3D du mouvement.

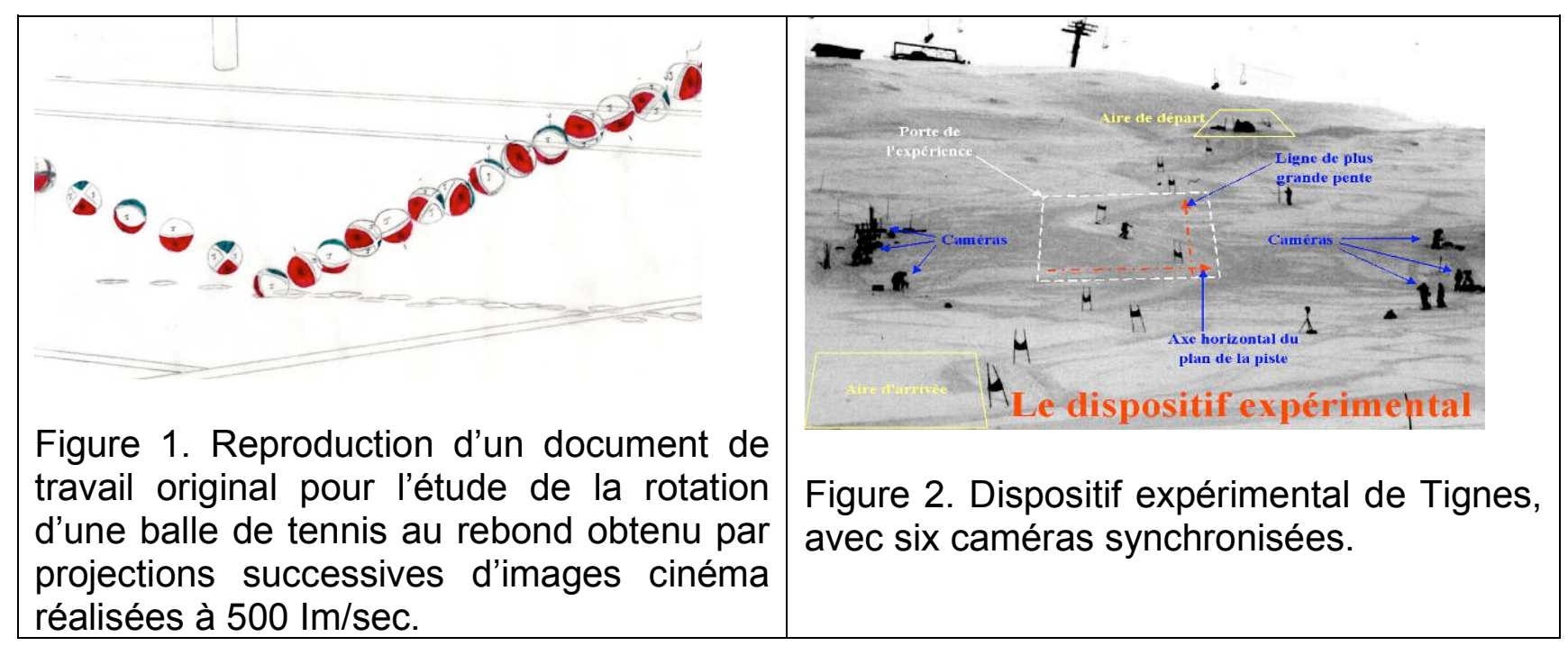

\section{Contractualisation et recherches finalisées}

S'inscrivant dans un cadre contractuel, les recherches ont été développées en relation avec les acteurs du monde sportif. Des partenariats ont ainsi été établis avec les fédérations sportives (tennis, tennis de table, ski nautique, athlétisme...) ou leurs cadres techniques et entraîneurs, les institutions (INSEP, AFNOR), les équipes universitaires, les industriels.

C'est ainsi que les modèles de trajectoires, de rebonds, et de chocs des balles en rotation, ont été validés par les réponses données aux problèmes issus directement des pratiques sportives. 
Au tennis, par la catégorisation des "coups » et des effets de balle, la production d'un lexique commun aux entraîneurs fédéraux et l'amélioration de la formation des cadres techniques de la Fédération Française de Tennis.

Au tennis de table, les mêmes modèles, adaptés à l'activité, ont apporté aux instances fédérales une aide à la décision concernant des modifications des règles du jeu (hauteur du filet, poids et diamètre de la balle).

Plus particulièrement, le modèle du rebond, issu des demandes du milieu industriel ou institutionnel a permis la conception et la mise au point de surfaces synthétiques nouvelles et la définition d'une nouvelle norme AFNOR caractérisant ces sols ainsi que la construction et la diffusion d'un appareil de mesure de ces caractéristiques in situ.

Des recherches se sont aussi développées sur l'analyse du mouvement d'activités sportives complexes. II s'agissait de rechercher par la production de modèles, des indicateurs (observables) destinés à l'entraînement (aide à la décision, optimisation de la performance) et à la formation (contenus).

Ainsi, dans les questions posées par les entraîneurs sur l'optimisation des performances, les modélisations du saut à la perche (1988, figure 3), ou du saut à ski nautique (1989), par la production de connaissances nouvelles, ont fait naître de nouveaux indicateurs de la performance.

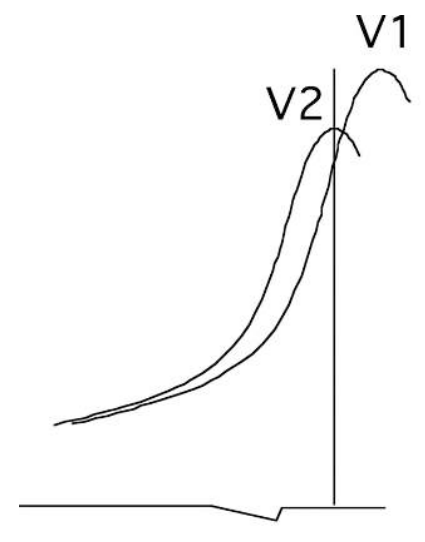

$$
\mathrm{V} 1>\mathrm{V} 2
$$

Figure 3. Trajectoires du CG pour deux vitesses différentes au décollage (en saut à la perche)

La méthodologie de départ, centrée sur le recueil de données cinématiques ou dynamiques, s'est largement ouverte à d'autres méthodes d'investigations (entretiens, analyse des discours...). 
D'autres travaux se sont attachés ainsi, à modéliser plus généralement l'activité ellemême ; au tennis de table ou au saut à ski nautique, des recherches ont mis en relation des contraintes mécaniques et physiques de l'activité, par une analyse et une modélisation des régulations spatio-temporelles du joueur ou du sauteur $(1989,1990)$.

De même, dans le domaine de la formation des entraîneurs, en articulant une analyse cinématique et dynamique de virages de slalom géant en compétition et des entretiens avec les entraîneurs, la thèse soutenue par François Roux (2000) sous la direction d'Alain Durey, s'est intéressée à la modélisation de la technique du virage en slalom géant pour permettre la conception de nouveaux outils de lecture pour les entraîneurs.

\section{Conclusion}

On peut mesurer à l'exposé rapide de ces quelques résultats, l'impact des travaux d'Alain Durey dans le domaine des APS, tant au niveau de l'analyse et de la modélisation des activités, que de celui de la transformation des pratiques, de l'entraînement et de la formation.

Je ne peux terminer ce propos sans évoquer l'enseignant et le formateur qu'était Alain Durey.

Engagé dans l'encadrement de l'option APS du DEA de Didactique des Disciplines puis du DEA Enseignement et Diffusion des Sciences et des Techniques de l'ENS Cachan, mais aussi dans l'enseignement de la physique dans la filière STAPS en DEUG, et dans les stages de formation des cadres techniques du Ministère Jeunesse et Sport, il s'est toujours efforcé de trouver les meilleurs outils pour un enseignement adapté à la population concernée.

Son ouvrage, Physique pour les sciences du sport, destiné aux étudiants STAPS, est une illustration forte de la complémentarité enseignant/chercheur puisque les concepts de la physique y sont explicités et les activités physiques et sportives étudiées en intégrant de nombreux résultats des recherches menées.

Enfin, qu'il me soit permis de dire combien toutes ces années passées avec lui, à " décortiquer » toutes ces APS, pour mieux les comprendre, pour redistribuer à la communauté les connaissances nouvelles, ont été source d'une richesse intellectuelle mais aussi et surtout humaine très intense. 


\section{Références}

Durey, A. (1997). La physique pour les sciences du sport. Paris : Masson.

Durey, A. (1994). Essai de caractérisation de quelques recherches technologiques en APS. Dossiers EPS, 19, 17-27.

Durey, A. (1987). Vers des activités didactiques de mise au point de modèles de physique avec des micro-ordinateurs. Exemples de trajectoires, frappes et rebonds de balles en rotation. Thèse de l'Université Paris 7 .

Alain Durey a dirigé plusieurs thèses dans le domaine des APS

Robin, Jean-François (1998). Spécificité, structure et sens de savoirs pour enseigner la gymnastique : étude de quatre leaders de théories didactiques.

Catteau, Alain (2001). Variation des paramètres du cycle de bras en crawl en fonction des conditions d'exercice et des niveaux d'expertise.

Roux, François (2000). Actualisation des savoirs technologiques pour la formation des entraîneurs de ski alpin de compétition.

Gérard, Jean-Paul (2003). Modélisation de la course de vitesse chez l'enfant : contribution à l'amélioration de l'habileté de lecture du mouvement par l'enseignant.

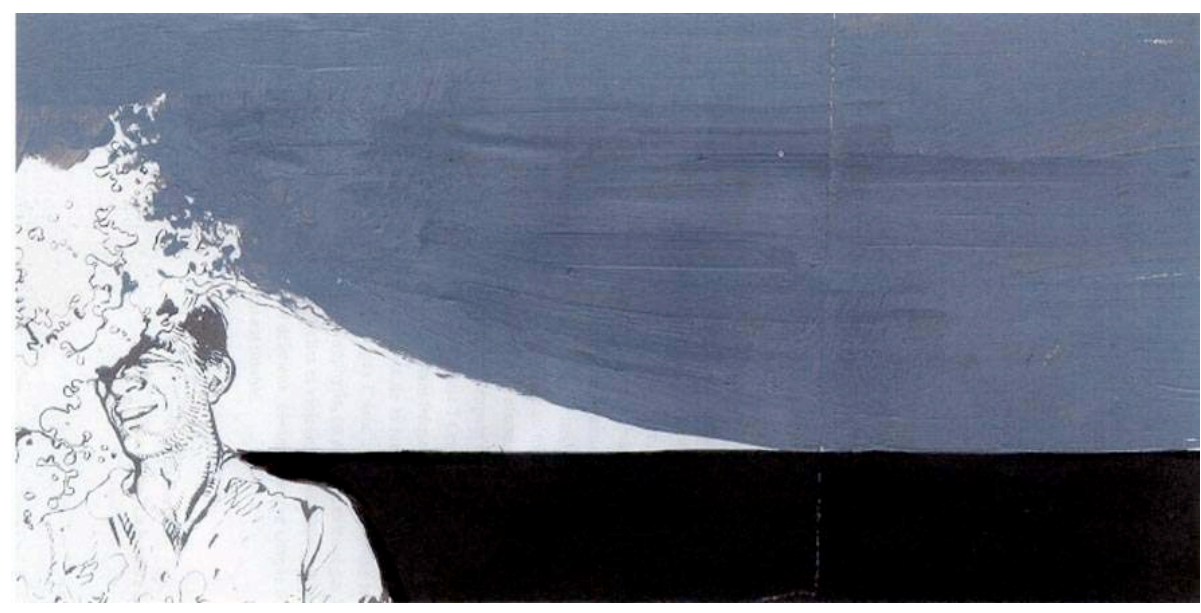

Casalegno 